\title{
Some combinatorial and spectral properties of Baxter operators and related linear transformations
}

\author{
Nguyen-Huu-Bong
}

The thesis investigates some combinatorial and spectral properties of Baxter operators, that is, of bounded linear operators $T$ on a Banach algebra $A$ with identity $e$, satisfying

$$
T x \cdot T y=T(T x \cdot y+x \cdot T y-\theta x y)
$$

identically for all $x$ and $y$ in $A$, the parameter $\theta$ being assumed to be a central element of $A$. As far as combinatorial properties are concerned, we restrict ourselves to the case $\theta=e$, when $T$ is called a summation operator. The reason is that the summation identity lends itself more readily to computational manipulations, as it does not involve any parameter.

We reserve part of our investigation into the spectral properties for general Baxter operators as defined above. Here, we have to impose certain conditions on the parameter $\theta$, with a view to elicit some connection which appears to exist between Baxter operators and averaging operators, the latter being defined by

$$
T(x \cdot T y)=T x \cdot T y=T(T x \cdot y)
$$

for all $x, y$ in $A$.

First we give a detailed historical survey of the whole situation; we also obtain some simple results, which constitute part of the motivation for part of the investigation proper. We then consider the combinatorial

Received 23 November 1970. Thesis submitted to Monash University, April 1970. Degree approved, September 1970. Supervisor: Professor J.B. Miller. 
problem. Through the use of inner derivation, defined on $B(A)$, the algebra of all bounded linear operators on $A$, and simple finite induction arguments we derive several combinatorial identities, both known and new, some involving Stirling numbers of the second kind.

Next we seek to determine the minimal polynomial associated with a summation operator $T$. It turns out that this is an immediate consequence of some results due to J.B. Miller and A.E. Taylor. We then consider some properties of a class of summation operators generated by a given summation operator $T$. The method used, via a suitably defined holomorphic function, which maps summation operators to summation operators, is both combinatorial and analytical.

Finally we consider the case of a general Baxter operator $T$. On imposing certain restrictions on $\theta$ we find that it is possible to obtain a formula for the resolvent of $T$ and some other spectral properties which bear close resemblance to those of an averaging operator. We also consider the cases when the analysis of $T$ can reduce to that of a summation operator. The reason is that more is known about averaging and summation operators than about the general Baxter operator itself. The results obtained indicate that, in general, there is no definite criterion that would allow us to state categorically that averaging operators are special cases of Baxter operators, or vice versa. 